\title{
A METHOD FOR IDENTIFYING NON-GAUSSIAN PARAMETRIC MODEL WITH TIME-VARYING COEFFICIENTS
}

\author{
Minfen Shen* Rong Song* K. H. Ting** Francis H. Y. Chan** \\ *Science Research Center, Shantou University, Guangdong 515063, China. Email: mfshen@stu.edu.cn \\ ** Department of Electronic Engineering, Hong Kong University, Hong Kong. Email: fhychan@eee.hku.hk
}

\begin{abstract}
A method for identifying non-Gaussian AR model with time-varying parameters is addressed. The proposed approach is based on the application of higher-order spectra (HOS) and wavelet analysis. To solve the problem and identify the characteristics of the time-varying linear system, a time-varying parametric model is proposed as a non-Gaussian AR model. The model coefficients that characterize the time-varying system are the functions of time and can be represented by a family of wavelet basis functions, having the invariant basis coefficients. This method can well track the changes of the model coefficients. The experimental results show the effectiveness of the proposed approach.
\end{abstract}

\section{INTRODUCTION}

Many methods for system identification and parameters estimation are exclusively based on the assumption of stationarity and Gaussinarity of the underlying processes. In spite of its many successful applications, aforementioned methods do not fulfill the nonstationary applications. Now more and more growing focus is put on the nonstationary environments, whose nonstationarity are closely related with. many physical world. The instantaneous information of real signals and systems are hard to be identified and predicted. There are many useful methods applied to nonstationary physical situations.

The most popular approach to estimate the nonstationary signals is to employ an adaptive algorithm and assume that the change of the signals is shown in [2]. Marc Lavielle solved the problem by presuming that the process is locally stationary over a relatively short time interval but globally nonstationary. Then we look on this kind of signals as piece-stationary signals and the most important thing is to find the instants of change [3-4]. More people construct the nonstationary models directly: Satoru Goto present cumulant-based methods for time-varying AR model parameters estimation $[5,6,8]$, but this method is limited in certain AR parameters. A novel Bayesian formulation is developed to identify the system parameters and estimate the models. Other report [7] uses a wavelet basis for the identification of time-varying (TV) system, and TV parameters can be expanded onto a finite set of wavelet basis sequences. Its flexibility in capturing the system's characteristics at different scales is at the cost of computational complexity. Several papers [1][6][7] involve the basis function. With the application of basis vectors such as Legendre polynomials and Fourier series, the TV model can be represented by a family of basis vectors, and the basis coefficients are invariant. We combine wavelet basis functions and the higher-order statistics, and propose a new method to estimate the coefficients of time-varying AR model, which is better than former methods. Section 2 of this paper introduces the parametric model of our method. Section 3 represents the experimental results and compares the method with Fourier method. Finally, some simulations are demonstrated.

\section{THE BASIC SCHEME}

We build a TV linear system or TV AR(P) model to extract the feature of the characteristics of the nonstationary signal. When $X=\left(X_{1}, X_{2}, \cdots X_{n}\right)$ is a nonstationary real process, the TV parameters of the model is employed that can be described by the following difference equation:

$$
x(n)=-\sum_{k=1}^{P} a_{k}(n) x(n-k)+v(n)
$$


where $v(k)$ is an independent identically distributed (i.i.d.) stationary, non-Gaussian process, with zero-mean and a finite nonzero cumulant. $a_{k}(n)$ are the TV parameters, which can be represented by a linear combination of a number of known functions:

$$
a_{k}(n)=\sum_{j=0}^{q} a_{k j} u_{j}(n)
$$

where $u_{j}(n)$ is the orthonormal basis functions. The TV AR coefficients are represented on the space spanned by the basis functions. TV AR coefficients are constants in this space. We can get $a_{k}(n)$ if we know the coefficients of the functions since the functions are all known. The model can be described as

$$
\begin{aligned}
& x(n)=-\sum_{k=1}^{p} a_{k}(n) x(n-k)+v(n)= \\
& \sum_{k=1}^{p}\left[\sum_{j=0}^{q} a_{k j} u_{j}(n)\right] x(n-k)+v(n)
\end{aligned}
$$

or

$$
X(n)=-\sum_{k=1}^{p} A(k) \cdot X(n-k)+V(n)
$$

where

$$
\begin{gathered}
X(n)=\left[u_{0}(n) x(n), u_{1}(n) x(n), \cdots \cdots, u_{q}(n) x(n)\right]^{T} \\
V(n)=\left[u_{0} v(n), u_{1} v(n), \cdots \cdots, u_{q} v(n)\right]^{T} \\
A(k)=\left[u_{0} a_{k}, u_{1} a_{k}, \cdots \cdots, u_{q} a_{k}\right]^{T}
\end{gathered}
$$

where

$$
a_{k}^{T}=\left[a_{k 0,} a_{k 1,}, \cdots, a_{k q}\right]^{T}
$$

The expression (4) can be evolved into a system of $q+1$ equations:

$$
\begin{gathered}
u_{0}(n) x(n)=-\sum_{k=1}^{p} u_{0}(n) a_{k}^{T} \cdot X(n-k)+u_{0}(n) v(n) \\
u_{1}(n) x(n)=-\sum_{k=1}^{p} u_{1}(n) a_{k}^{T} \cdot X(n-k)+u_{1}(n) v(n) \\
\vdots \\
u_{q}(n) x(n)=-\sum_{k=1}^{p} u_{q}(n) a_{k}^{T} \cdot X(n-k)+u_{q}(n) v(n)
\end{gathered}
$$

We also know that the mth order cumulants sequence of $X(n)$ satisfies the following recursive equation [5]:

$$
\sum_{k=0}^{P} A(k) C_{m, X}\left(\tau_{1}, \cdots ; \tau_{m-2}, \tau-k\right) \equiv 0, \quad \tau>0
$$

We assume $m=3$, then (5) can be changed into:

$$
\sum_{k=1}^{p}\left[\begin{array}{c}
u_{0}(n) a_{k}^{T} \\
u_{1}(n) a_{k}^{T} \\
\vdots \\
u_{q}(n) a_{k}^{T}
\end{array}\right]\left[\begin{array}{c}
c_{j, 1,1}, \cdots c_{j, q+1,1} \\
c_{j, 1,2}, \cdots c_{j, q+1,2} \\
\vdots \\
c_{j, 1, q+1}, \cdots c_{j, q+1, q+1}
\end{array}\right] \equiv 0
$$

where $c_{j, 1,1}=E\left[x_{j}(n) x_{1}\left(n+\tau_{1}\right) x_{1}(n+\tau-k)\right]$ and $x_{j}(n)$ is the jth element of $\mathrm{X}(\mathrm{n})$

\section{WAVELET BASIS FUNCTION}

A wavelet orthonormal basis of the usual Lebesque square integrable function space $L^{2}$ satisfies the following formula:

$$
<\psi_{k, n}, \psi_{l, m}>=\delta_{k l} \cdot \delta_{n m}, \quad k, n, l, m \in Z
$$

if $f \in L^{2}(R)$, we can get:

$$
f(x)=\sum_{j, k \in Z} c_{j, k} \psi_{j, k}(x)
$$

Some kinds of wavelet basis can be chosen to represent the $A R$ coefficients, such as Harr and Daubechies basis, because of their good performance in transient change. For time series with sharp jumps or steps, one would choose a boxcar-like wavelet function such as the Harr and Daubechies basis. On the other hand, we would choose a smooth function such as a dumped cosine for smoothly varying time series. It is the Fourier transform which is a special case of a wavelet transform that has basis vectors defined by trigonometric functions--sine and cosine. So we choose Harr basis and Fourier basis respectively in the following simulations.

It is notable that Harr basis has interesting behavior as being capable of capturing the global as well as the 
local behavior of the TV coefficients. It consists of scaled and translated versions of a single function.

\section{RESULTS AND DISCUSSION}

We assume that the $A R$ order $p=2$, then:

$$
x(n)=a_{1}(n) x(k-1)+a_{2}(n) x(k-1)+v(n)
$$

where $\mathrm{v}(\mathrm{k})$ is an independent identically distributed stationary, non-Gaussian process, whose variance $E\left[v^{2}(k)\right]=Q$ and $E\left[v^{3}(k)\right]=\beta \neq 0 .\left\{a_{1}(k)\right.$, $\left.a_{2}(k)\right\}$ are TV $\mathrm{AR}(2)$ model parameters changing abruptly in the following manner:

$$
\begin{aligned}
& a_{1}(k)=-1.5, a_{2}(k)=0.8 \\
& k \in[1, N / 4] \cup[N / 2+1,3 N / 4], \\
& a_{1}(k)=-0.9, a_{2}(k)=0.2 \\
& k \in[N / 4+1, N / 2] \cup[N 3 / 4+1, N],
\end{aligned}
$$

The TV process is generated with $\mathrm{N}=6000$ samples in Fig.1 and 2, which are shown by the rectangle blue wave. Both Fourier basis and Harr basis are used to estimate the model parameters, and compare the results with the original values.

Both Fig.1 and Fig.2 depict the true $\operatorname{AR}(2)$ parameters $a_{1}(k)$ and $a_{2}(k)$, and the estimated results based on Fourier basis and Harr basis, respectively. It is important to note that the estimation based on Harr basis responds rapidly and precisely to the parameters' time-variances. This finding highlights the capacity of wavelets to identify time-variances that may have physiologic relevance and retains parametric identity even though model coefficients vary continuously over the data segment studied. It can be seen from the results that Harr basis is much better than Fourier basis for tracking the changes of the model coefficients. We can get the same result by the error criterion which is estimated by minimizing a penalized contrast function of the form [3]:

$$
\begin{aligned}
& d_{0}\left(x_{1}, x_{2}, \cdots, x_{N} ; a_{1}, a_{2} \cdots a_{p}\right)= \\
& \sum_{i=P+1}^{N}\left[X_{i}-\sum_{j=1}^{p} \hat{a}_{j} \hat{X}_{i-j}\right]^{2} / N
\end{aligned}
$$

The error of wavelet basis with $d_{01}=1.7332$ is less than the error of Fourier basis with $d_{02}=2.0256$, which reflect that the wavelet basis is more suitable than the conventional method in the identification of the TV linear systems.

\section{CONCLUSION}

The aim of the proposed method was to investigate the problem of the identification of the time-varying linear systems described by an non-Gaussian AR model. The AR model parameters that characterize the time-varying system are functions of time and can be represented by a family of wavelet basis functions. A comparison between wavelet basis and Fourier basis of cumulants-based method is also given. The results in the presented method show the applicability and the effectiveness of the procedures, while some signal processing techniques is needed to apply to minimize the estimated error.

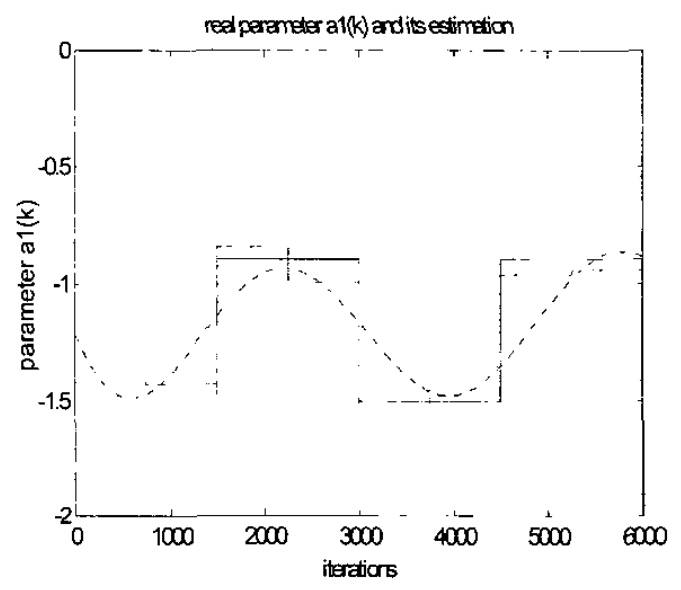

Fig. 1 The estimated result of the TV $a_{1}(k)$. The estimated result of Fourier basis is shown as the dotted line, and the estimated result of Harr basis is shown as the solid line. 


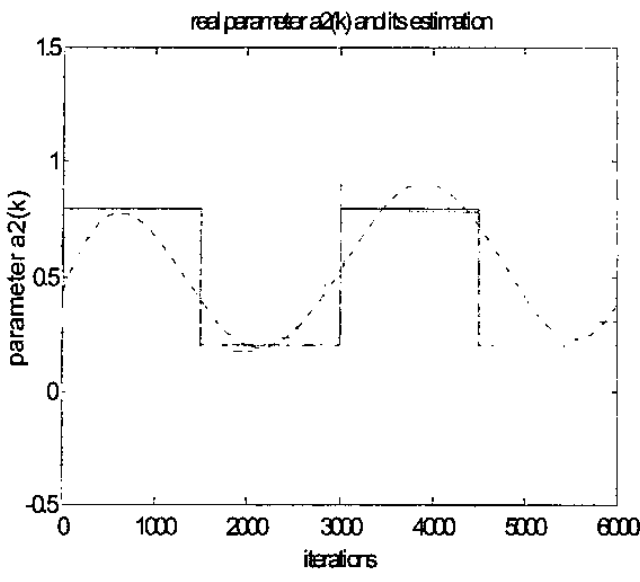

Fig. 2 The result of the estimation of the TV $a_{2}(k)$. The estimated result based on Fourier basis is shown as the dotted line, and the estimated result based on Harr basis is shown as the solid line.

\section{ACKNOWLEDGEMENTS}

The research is in part supported by the National Natural Science Foundation of China (60271023), the Key Foundation of Guangdong Natural Science Foundation 2002 (021264) and the Key Foundation of Education Department of China (02110).

\section{REFERENCES}

[1] J. J. Rajan, Peter J. W. Rayner "Generalized Feature Extraction For Time-varying Autoregressive Models", IEEE Trans. on Signal Processing. vol. 44, No. 10, October 1996.

[2] D. Aboutajdine "Fast Adaptive Algorithms for AR Parameters Estimation Using Higher Order Statistics" IEEE Trans. on Signal Processing. vol. 44, No.8, Aug. 1996.

[3] Marc Lavielle "Optimal Segmentation of Random Processes," IEEE Trans. on Signal Processing. vol. 46, No.5, May 1998

[4] D. Geman, "Random fields and inverse problems in imaging," in Lecture Notes in Mathematics. New York: Springer -Verlag, 1990

[5] Lee M. Garth "A comparison of optimized higher-order spectral detection techniques for non-Gaussian signals," IEEE Trans. on Signal Processing. vol. 44, No.5, May 1996.
[6] M. Bakrim, D. Aboutajdine, and M. Najim, "New cumulant-based approaches for non-Gaussian time varying AR models," Signal Processing, vol. 39, nos. 1/2, pp. 107-115, Sept. 1994.

[7] M. K. Tsatsanis and G. B Giannakis. "Time-varying System Identification and Model Validation Using Wavelets." IEEE Trans. on Signal Processing, vol. 41,No.12, pp.3512-3523, 1993.

[8] M. Martone "Non-Gaussian Multivariate Adaptive AR Estimation Using the Super Exponential Algorithm," IEEE Trans. on Signal Processing, vol. 44, No.10, Oct. 1996.

[9] G. Thonet, Jean-Marc Vesin "Stationarity Assessment with Time-varying Autoregressive Modeling" Signal Processing Laborator, pp. 3721-3724, 1997. 